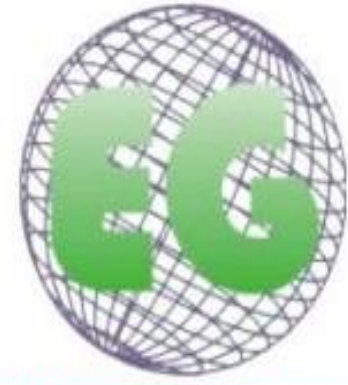

ISSN 1695-6141 $N^{\circ} 46$

\title{
Prácticas alimentarias del alumnado de secundaria durante la jornada escolar en Andalucía asociadas a la oferta del entorno
}

Dietary habits of secondary school students in Andalusia during school time in relation to food stores in the environment

\section{*García-Padilla, Francisca María *González-Rodríguez, Angustias **Martos- Cerezuela, Ildefonso ***Liébana Fernández, José Luis ****Melo Salor, Juan Antonio y Grupo del Proyecto ANDALIES^}

\author{
*Departamento de Enfermería. Universidad de Huelva. E-mail: fmgarcia@uhu.es **Unidad de \\ Gestión Clínica Ciudad Jardín. Distrito Sanitario Málaga. ***Unidad de Gestión Clínica Martos. Distrito \\ Sanitario Jaén-Sur. ****Unidades de Gestión Clínica Montequinto, Olivar de Quinto y El Campo de las \\ Beatas. Área de Gestión Sanitaria Sur de Sevilla. España.
}

http://dx.doi.org/10.6018/eglobal.16.2.239881

\section{RESUMEN}

Fundamento: El análisis del contexto ambiental es imprescindible para estudiar las prácticas alimentarias en adolescentes. Se describen las prácticas alimentarias durante la jornada escolar del alumnado usuario de los comercios de alimentos circundantes a los centros de educación secundaria en Andalucía y las características de los mismos.

Métodos: Estudio descriptivo transversal, sobre una muestra de 8068 estudiantes y 95 centros de secundaria. Muestreo aleatorio polietápico por conglomerado: estratificado por provincia y tamaño de hábitat y sistemático por aulas. El tipo de establecimiento, oferta alimentaria, distancia y visualización desde el centro y demanda del alumnado se realizó mediante observación directa y entrevistas, y las características socio-demográficas y las prácticas alimentarias a través de cuestionario.

Resultados: Los centros tienen establecimientos cercanos con oferta alimentaria dirigida al alumnado $(72,63 \%)$. Predominan bares y cafeterías (55), tiendas de comestibles (54) y quioscos de chucherías (23). La oferta de chucherías, bollería y bocadillos se constata en todos los establecimientos. El $25,73 \%$ del alumnado compra su merienda escolar en estos comercios, encontrando relaciones significativas en el consumo de golosinas y paquetes de fritos envasados frente al alumnado que no sale del instituto durante la hora del recreo.

Conclusiones: El número y las características de la oferta de los establecimientos circundantes a los centros educativos obligan a profundizar en la influencia sobre las prácticas alimentarias del alumnado para establecer medidas de salud pública que controlen la oferta insalubre detectada. 
Palabras clave: Educación secundaria; promoción de la salud; educación alimentaria, hábitos alimentarios, adolescentes.

\section{ABSTRACT}

Background: The analysis of the environmental context is indispensable to study the eating habits in adolescents. Feeding practices during the school day the students user surrounding grocery stores of secondary schools in Andalusia and their characteristics are described.

Methods: Descriptive cross-sectional study of 8068 students and 95 secondary schools. Multistage cluster sampling: Stratified random sampling by province and population size and systematic for classrooms. The analysis of the type of store, food supply, distance and visualization from the school and students' demands was conducted by direct observation and interviews. The socio-demographic characteristics and feeding practices were got through questionnaire.

Results: The schools are surrounded by shops in which the food supply is aimed at students $(72,63$ $\%)$. Pubs and cafeterias (55), grocery stores and tuck shops predominate. The presence of sweets, processed baked goods and sandwiches is confirmed in all the stores. $25.73 \%$ of students buy their school meals in these shops. There are statistically significant relationships between the consumption of sweets and fried packaged packages and to go out the high school during the playground.

Conclusions: The number and characteristics of the stores surrounding the schools require studying its influence over students eating habits in order to establish public health measures to control the detected unhealthy supply.

Keywords: Diet; Secondary Education; health promotion; food education; eating habits, teenager.

\section{INTRODUCCIÓN}

A partir de la creciente preocupación por el estudio de las relaciones de la alimentación con la salud en la población juvenil española, múltiples estudios evidencian déficits alimentarios en esta etapa ${ }^{1,2}$. La población infantil y juvenil es, por definición, una población sana, aunque a consecuencia de los estilos de vida inadecuados están emergiendo serios problemas de salud. La alimentación de los escolares está amenazada por un creciente número de variables que están incidiendo en la abundante oferta de productos no recomendados en una alimentación saludable $^{3}$. Diversos estudios sugieren que los problemas derivados de los hábitos alimentarios de adolescentes y adultos jóvenes en Europa siguen pautas similares, considerando las desigualdades geográficas y socioeconómicas específicas de cada persona, grupo y comunidad ${ }^{4}$.

La socialización de los niños/as y adolescentes se desarrolla en una parte importante del tiempo de su vida cotidiana en torno a los centros educativos. De ahí que, en el análisis de los factores que inciden en las prácticas alimentarias, sea necesario el estudio de la disponibilidad de alimentos en el entorno más cercano de los escolares ${ }^{5}$. La comprensión de la influencia del lugar representa un gran desafío de la investigación en salud, aunque la creciente prevalencia de obesidad a nivel mundial ha incrementado la investigación centrada en los factores del entorno ${ }^{6-9}$. Se ha sugerido la utilidad de estudios enfocados al contexto ambiental; cómo indagar acerca de las características de los barrios, lo que podría orientar sobre las intervenciones de prevención de la obesidad y la promoción de hábitos saludables en la población ${ }^{5}$. Las condiciones de acceso a los alimentos en la escuela, el hogar, los establecimientos comerciales son características del entorno que deben tenerse en cuenta a la hora de establecer las prioridades en las políticas de salud ${ }^{10}$. 
Los estudios que indagan sobre las relaciones del consumo alimentario de escolares y la oferta de los locales externos es muy prolífica en Estados Unidos ${ }^{8,11,12}$ y Australia $^{8}$. En Europa, aunque este objeto de estudio es incipiente, es reseñable el estudio IDEFICS ${ }^{13}$ dedicado al seguimiento de los riesgos ambientales asociados al sobrepeso infantil. Aunque en él no se ha podido demostrar que las tiendas de alimentos minoristas, que se agrupan en torno a las escuelas, favorecen un mayor consumo en los/as niños/as pequeños/as. Sin embargo, investigaciones desarrolladas en Estados Unidos han establecido asociaciones positivas ${ }^{14}$.

En concordancia con los planteamientos de estos autores es relevante el estudio de las tipologías de los locales periféricos y las razones de la ubicación en torno a los centros educativos a los que pertenecen los escolares. La disponibilidad de locales periféricos se consideraría un factor del nivel meso, aspecto analizado en estudios dedicados a establecer qué tipos de entornos urbanos propician el desarrollo de la obesidad $^{5}$. Además del fácil acceso de alimentos saludables, otras características de los barrios a considerar como determinantes, son la calidad del medioambiente físico, el nivel socioeconómico de la zona y las desigualdades en salud. En este sentido, estudios realizados en Estados Unidos demuestran que el acceso a tiendas que venden alimentos saludables es peor en los barrios de bajos ingresos ${ }^{5}$. Bajo este enfoque, es relevante el desarrollo de estudios que indagan sobre las influencias del entorno escolar en las prácticas alimentarias del alumnado desde una perspectiva más amplia ${ }^{15}$.

En España, resulta más complejo conocer la oferta y el consumo alimentario de los escolares adquiridos en los comercios de la periferia de los centros de secundaria ya que, hasta el momento, este aspecto posee un escaso o inexistente interés institucional en nuestro país. Aunque en los últimos años se está haciendo un esfuerzo legislativo por llevar a cabo un control interno de los productos alimentarios consumidos y/o adquiridos en los centros educativos con la promulgación de la Ley 17/2011, de Seguridad Alimentaria y Nutrición ${ }^{16}$, su implantación y desarrollo deja mucho que desear, al menos en los centros de educación secundaria de Andalucía ${ }^{17}$. Dada la inexistencia de investigaciones en nuestro ámbito, el estudio que se presenta tiene el objetivo de describir y categorizar los comercios ubicados en las cercanías de los centros de enseñanza secundaria, cuya oferta incluya alimentos orientados al consumo del alumnado, caracterizar al alumnado que hace uso de dichos establecimientos e identificar posibles influencias en sus prácticas alimentarias. Este artículo forma parte de un estudio más amplio realizado en Andalucía denominado Proyecto ANDALIES.

\section{MATERIAL Y MÉTODOS}

Se ha realizado un estudio observacional descriptivo durante los años 2011-2012 sobre los establecimientos comerciales situados en la periferia de una muestra de 95 Institutos de Educación Secundaria (IES) y una muestra de 8068 estudiantes de Andalucía. Los centros fueron seleccionados mediante un muestreo aleatorio estratificado con afijación proporcional por provincia y tamaño de hábitat, para un error muestral de $\pm 10 \%$ y un Nivel de Confianza (NC) del $95,5 \%$. La segunda unidad de análisis fue el alumnado, elegido mediante un muestreo sistemático de las aulas de los IES previamente seleccionados. El error muestral fue de $\pm 2 \%$ para NC 95,5\%. Las variables estudiadas de los locales externos han sido: tipo de establecimiento, oferta alimentaria, distancia al centro, visualización desde el centro y demanda del alumnado. La recogida de datos se efectuó mediante la observación directa de los 
locales, con una guía de observación pilotada previamente en dos centros de la provincia de Huelva durante el año 2010 (Figura 1).

Figura 1. Guía de Observación: Estudio de locales comerciales externos para el abastecimiento de alimentos durante el horario escolar en las cercanías del centro educativo

Nombre del Centro Educativo

Población.

Persona

que

realiza

Provincia.

observación.

la

guía

de

Fecha en la que se realiza

Establecimiento $\mathrm{n}^{\circ}$ :

\begin{tabular}{|c|c|c|}
\hline \multirow[t]{5}{*}{ G2.1 } & \multirow{5}{*}{$\begin{array}{l}\text { Tipo de } \\
\text { establecimiento }\end{array}$} & $\square$ Quiosco \\
\hline & & Tienda \\
\hline & & $\begin{array}{l}\text { comestibles } \\
\square \text { Bar }\end{array}$ \\
\hline & & - Cafetería \\
\hline & & $\begin{array}{l}\text { Otros: } \\
\text { Especificar } \\
\text { tipo.............. }\end{array}$ \\
\hline
\end{tabular}

Observaciones del establecimiento:

Para la triangulación de la información, también se recogieron imágenes fijas y móviles, se entrevistó a las personas encargadas de estos establecimientos sobre la demanda de productos por parte del alumnado de secundaria y al equipo directivo del centro educativo sobre el conocimiento de la existencia de locales externos orientados al consumo del alumnado.

Las variables estudiadas del alumnado son: sexo, edad, provincia, tamaño de la población, tener cafetería el IES, desayunar en casa antes de ir al IES, tipo de desayuno, tipo de merienda escolar, consumo de dulces y/o bollería durante la jornada escolar, consumo de paquetes de fritos variados, consumo de golosinas durante la jornada escolar, realización de actividad física fuera del horario escolar, utilización de los locales externos al IES durante la hora del recreo, tipo de establecimientos que frecuenta.

El procesado y análisis de la información se ha realizado de dos formas: Los datos cuantitativos se han organizado y procesado mediante el paquete estadístico SPSS v. 18, calculándose la media, desviación típica, las frecuencias relativas y la chicuadrado para el análisis relacional; el análisis cualitativo de la información obtenida a través de la guías de observación, las imágenes y las entrevistas, se ha volcado en tablas para su categorización, realizándose un análisis documental.

\section{RESULTADOS}

El $72,63 \%$ de los centros educativos de Andalucía estudiados (69 IES) tienen en sus alrededores establecimientos cercanos que pueden abastecer de productos alimentarios al alumnado de secundaria. La provincia de Huelva es la que más IES 
tiene con este tipo de comercio en sus cercanías frente a Jaén o Granada que presentan menos (Tabla I). Se han registrado un total de 152 locales, cuya distribución provincial aparece en la Tabla I.

La variabilidad de comercios es amplia (24 tipos) por lo que se han agrupado en seis secciones para facilitar el análisis de la oferta alimentaria que realizan (Tabla II).

La sección 1 concentra a 54 locales, en la que se incluyen establecimientos cuya principal característica es la venta de productos de alimentación. En este grupo existe mucha diversidad en cuanto a tamaño y amplitud de la oferta de productos, aunque en el $42,1 \%$ de estos locales se constata una oferta abundante de chucherías, bollería, bocadillos a base de embutidos y refrescos.

La sección 2 representa el tipo de establecimiento más numeroso en nuestra comunidad. Los bares y cafeterías de los alrededores se contabilizan en 55 y unidos a los locales de un mismo sector suman 60. Aunque se observan en algunos negocios la existencia de bollería, algunas chucherías y bocadillos, la oferta principal es el desayuno típico: desayunos a base de café con leche, tostadas con paté y mantequilla y en algunos, zumo.

En el sector 3, encontramos la tradicional venta de churros y patatas fritas que en los casos registrados se ajustan a su perfil.

El grupo 4 aglutina a los comercios diana para la venta de todo tipo de chucherías. Se han contabilizado 23 quioscos, siendo de corte tradicional 20 y el resto bajo otras denominaciones aunque con un tipo de oferta convencional. La provincia de Huelva es la que acumula un mayor número de este tipo de locales estando presentes en el $71,42 \%$ de los centros de educación secundaria.

El sector 5 está encabezado por la panadería en la que se desdibuja su carácter tradicional con la introducción del punto de pan caliente de pan precocinado. En todos los negocios de este grupo se da la venta de productos como bollería, bocadillos y chucherías.

La medida de la distancia entre los IES andaluces estudiados y los locales identificados (Tabla I) determina que los más cercanos se encuentran a tan solo 10 pasos en Huelva y Córdoba, con una media interprovincial de 27,25 pasos. En general, Córdoba seguida de Cádiz son las provincias con establecimientos más cercanos en un mayor porcentaje de IES (50\% y $41,17 \%$, respectivamente). Sin embargo, en Jaén y Málaga los locales se encuentran a más de 150 pasos en la mayoría de los IES $(77,77 \%$ y $61,53 \%$, respectivamente).

Aunque el $65,79 \%$ de los locales no se pueden visualizar desde el centro educativo, son frecuentados por el alumnado y en ocasiones también por el profesorado, según declaran los regentes de dichos establecimientos y de los equipos directivos de los centros. 
Tabla I. Distribución provincial de establecimientos comerciales y distancia en pasos a los centros educativos

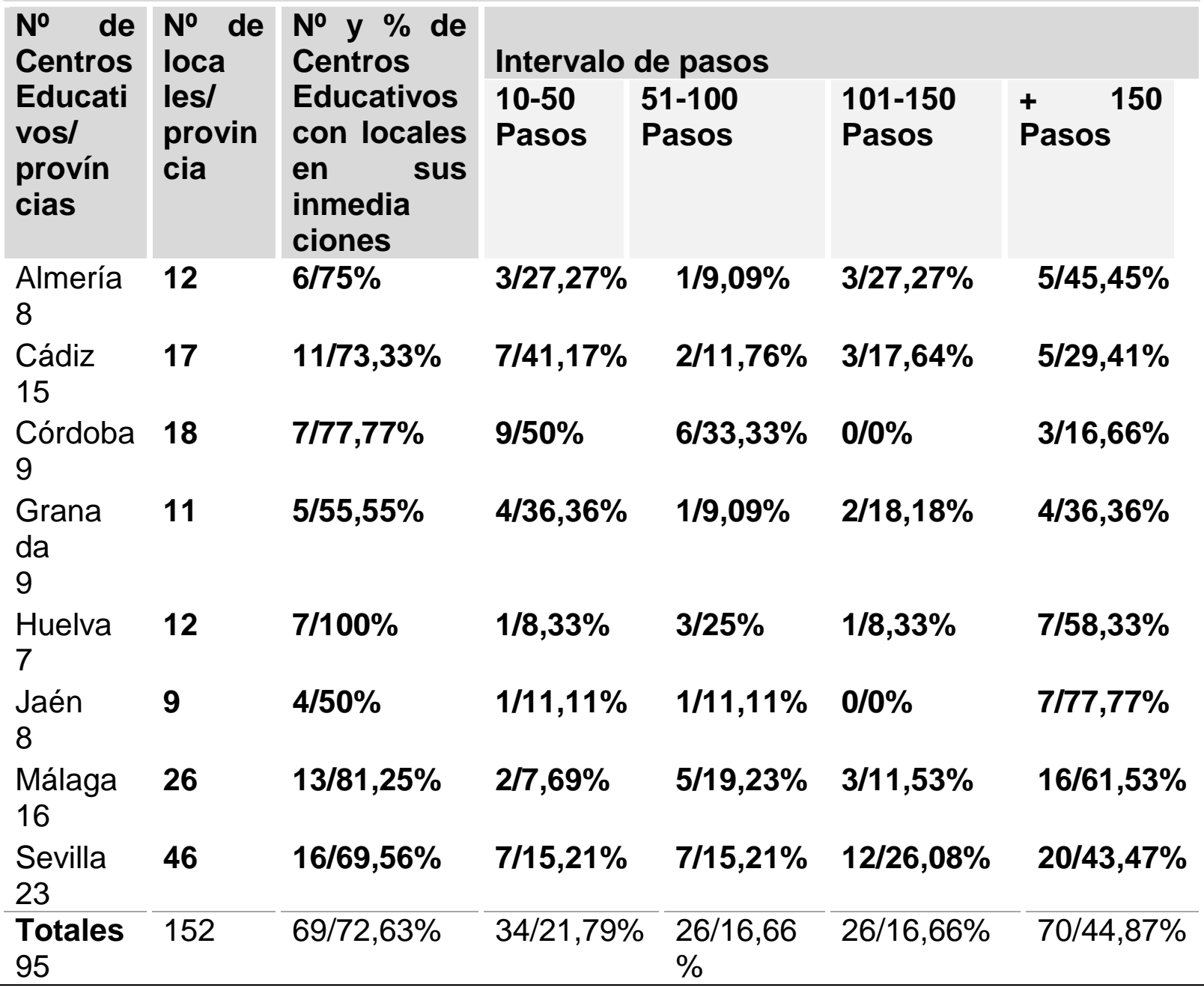

A través de las entrevistas, al equipo directivo, se determina que el $73,68 \%$ conoce la existencia de establecimientos externos al IES en los que se ofertan productos alimentarios y a los que el alumnado acude antes o durante el recreo, en el caso de los mayores de 18 años que pueden salir del centro. En algunas de las respuestas más representativas se tiene conocimiento además sobre el tipo de productos que adquieren como bocadillos, chucherías y bebidas, "el alumnado compra bastantes chucherías pero fuera del horario escolar" (P5C77) "Antes de entrar al IES pasan por algún establecimiento de alrededor a comprar chuches" (P7C72) "sí hay una panadería que les proporciona bocadillos de charcutería” (P6C47). Tan sólo un $12,63 \%$ de los directivos consultados ignora la presencia de estos locales.

El $25,73 \%$ del alumnado de secundaria adquiere su merienda escolar en algún establecimiento cercano al IES. Un 8\% aproximadamente salen siempre del IES durante la hora del recreo, un $31 \%$ no sale nunca y el $60,5 \%$ afirma salir a veces para comprar algún alimento.

Las características socio-demográficas y el patrón alimentario de los estudiantes que afirman o niegan salir del centro, se describe en la Tabla III y IV, respectivamente. Podemos destacar que la tipología de locales utilizado por el 34,55\% del alumnado, el quiosco para el 25,79\%, seguido de la tienda de comestibles (15,63\%), un supermercado $(14,22 \%)$ u otro tipo de establecimiento para el $9,78 \%$ del alumnado. 
De los que salen siempre del IES durante el recreo, mayoritariamente son de las provincias de Almería $(32,6 \%)$ y de Huelva $(20,2 \%)$, mientras que el alumnado de Sevilla es que sale del centro de forma más esporádica (29,8\% contesta a veces). Esto ocurre con más frecuencia en los IES de poblaciones con más de 100.000 habitantes $(38,2 \%$ y $28,2 \%)$. Salen fuera a pesar de existir cafetería en el $75,7 \%$ de los centros.

Tabla II. Tipos de locales cercanos a los centros de educación secundaria en Andalucía Variedad de Alme Cádiz Córdo Grana Huel Ja Mála Sevi Total establecimien ría ba da va én ga lla

tos

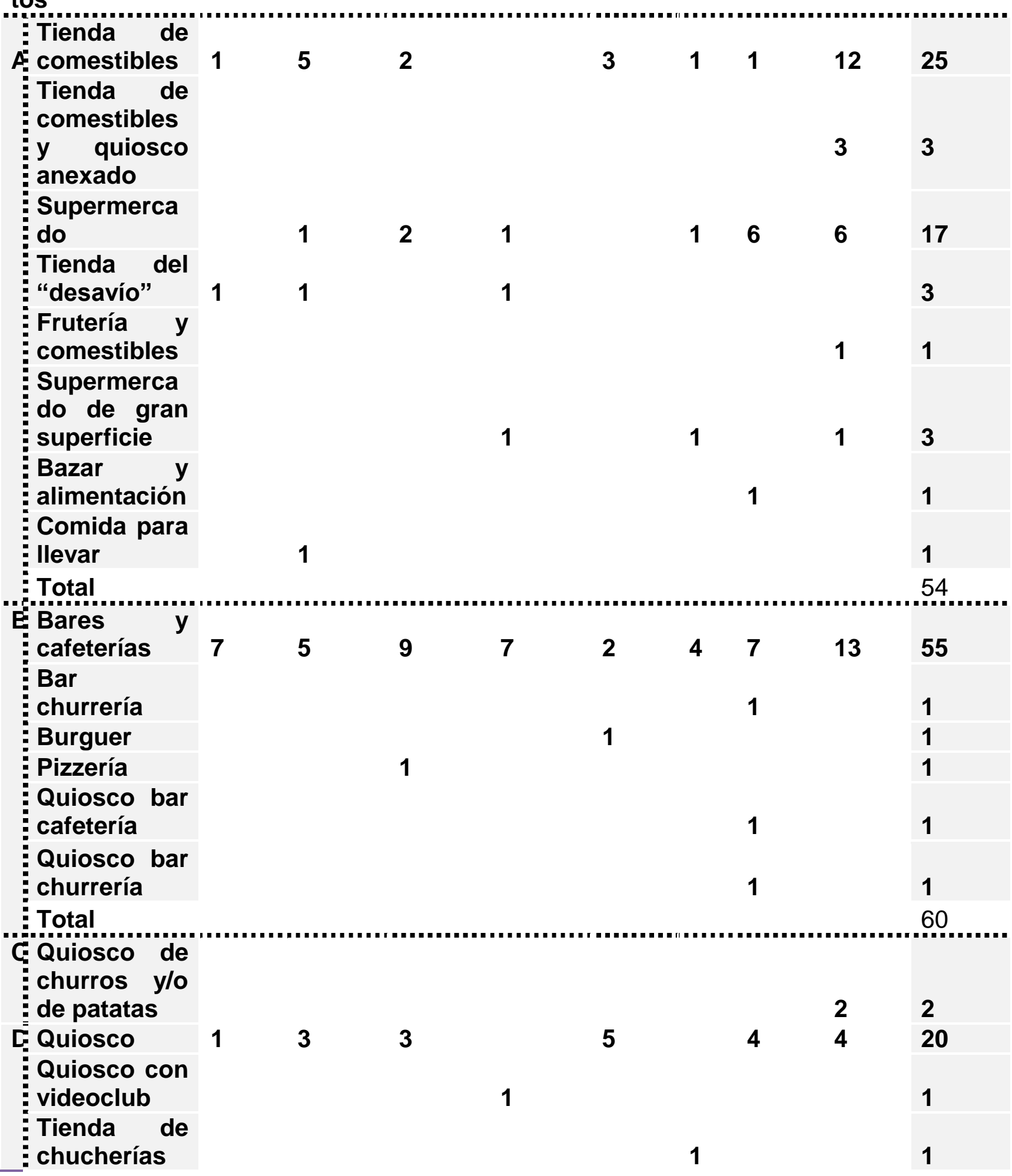




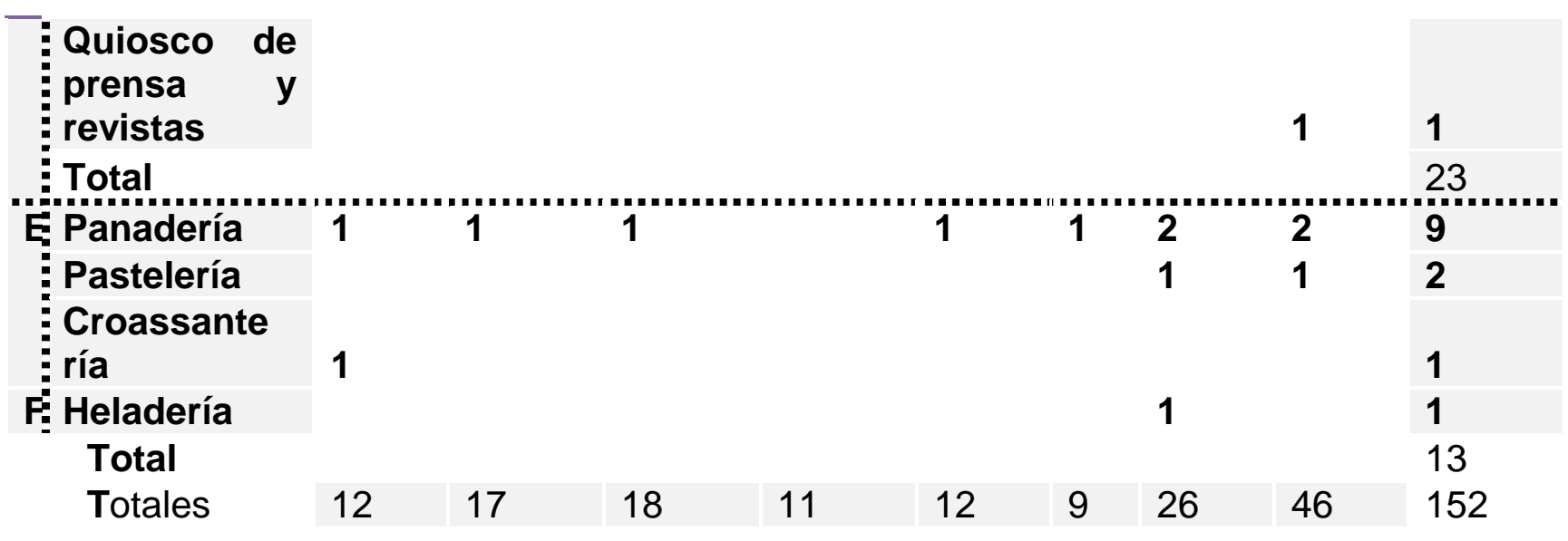

El $30,5 \%$ de este alumnado no desayuna en casa antes de ir al IES, y en el caso de desayunar, toman un medio desayuno con pan y/o cereal $(25,4 \%)$ o un desayuno líquido solamente $(22,1 \%)$. En el caso de la merienda, el $48,7 \%$ consume merienda tipo del alumnado de secundaria andaluz: Bocadillo acompañado de zumo comercial; $21,4 \%$ una merienda basada en algún líquido más Productos No Recomendables en una Alimentación Saludable (PNRAS); el 8,6\% sólo alimentos líquidos y un $8,4 \%$ añade a lo que merienda algún PNRAS. Por lo que $38,4 \%$ consume PNRAS.

En el análisis relacional es destacable las relaciones significativas encontradas entre las variables -Salir del IES durante el recreo para adquirir algún alimento- y variables como provincia, tamaño de la población, tener cafetería el IES, el tipo de desayuno, tipo de merienda y el consumo de golosinas (Tabla V).

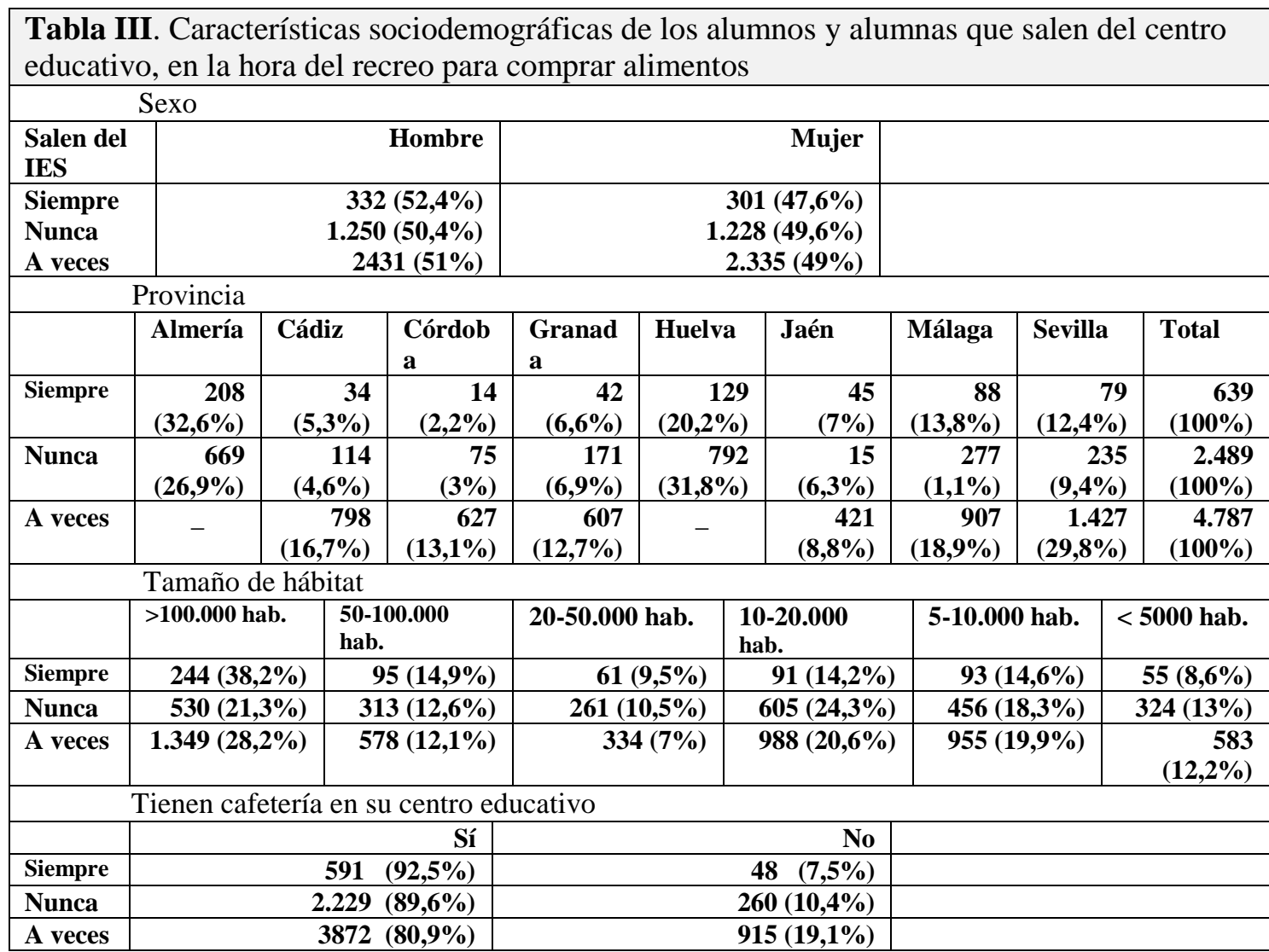

De tal forma que Almería y Huelva se alzan como las provincias en las que más sale el alumnado en la hora del recreo de forma significativa respecto a las demás; se 
suele dar más en poblaciones de más de 100.000 habitantes. Suelen salir en un mayor porcentaje en aquellos centros que carecen del servicio de cafetería. Respecto a las prácticas alimentarias, destacar que el alumnado que sale del IES consume significativamente dos tipos de desayuno: el compuesto por media porción de pan o cereal con un lácteo $(41,8 \%)$ y el desayuno consistente en un líquido únicamente $(29,2 \%)$.

Es reseñable el hecho de que el $81 \%$ de los estudiantes, que afirma tomar un desayuno de baja calidad nutricional, salgan del IES durante la hora de recreo para consumir algún producto alimentario. En relación a la merienda escolar, lo más frecuente y significativo es el tipo de merienda compuesto por un sólido (bocadillo) y un líquido (zumo comercial) para el $58,9 \%$ de los estudiantes que salen fuera del centro, merienda tipo del estudiante de secundaria andaluz.

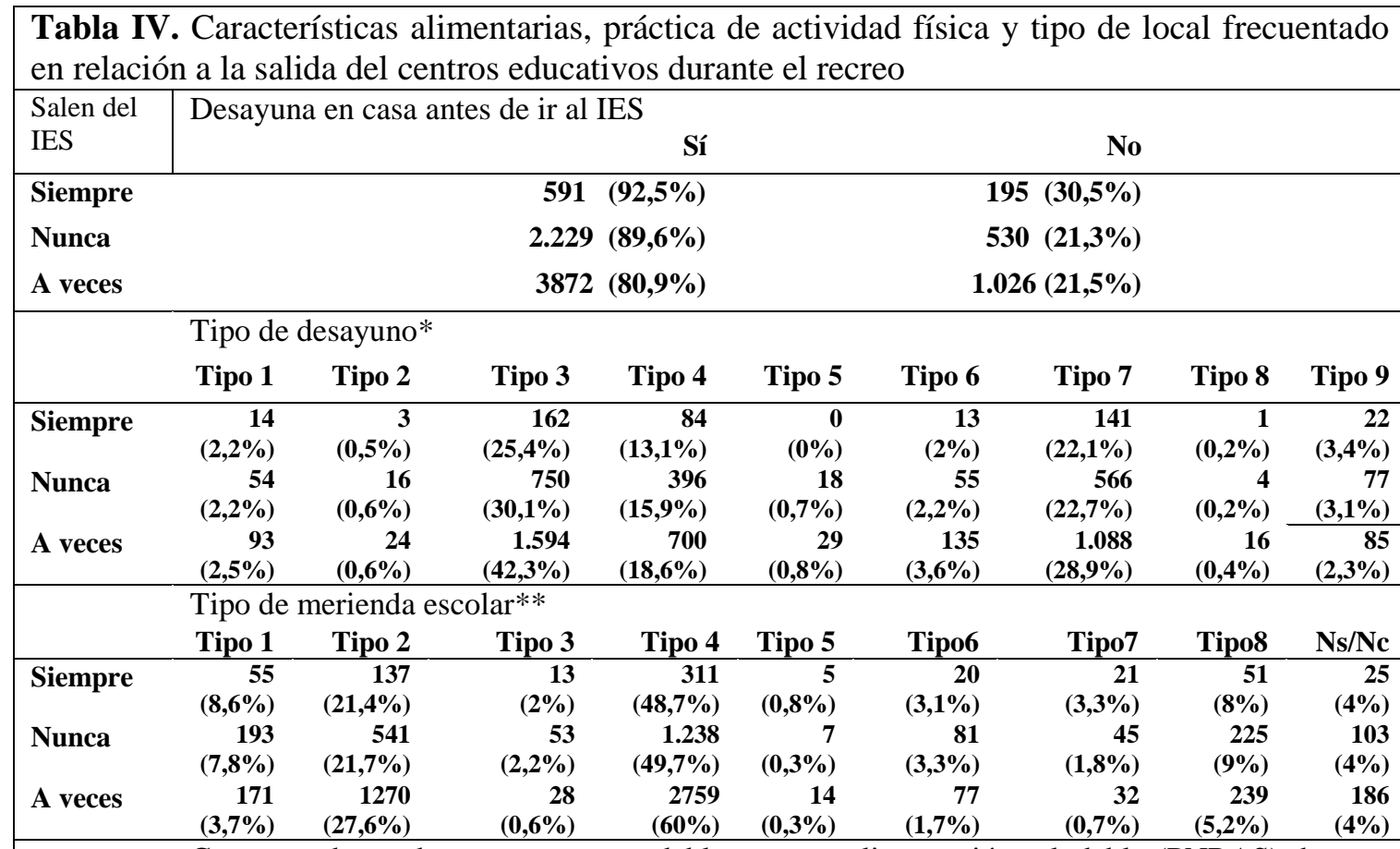

\begin{tabular}{|c|c|c|c|c|c|c|}
\hline & \multicolumn{6}{|c|}{$\begin{array}{l}\text { Consumo de productos no recomendables en una alimentación saludable (PNRAS) durante } \\
\text { la jornada escolar }\end{array}$} \\
\hline & \multicolumn{2}{|c|}{ Bollería } & \multicolumn{2}{|c|}{ Paquetes de fritos } & \multicolumn{2}{|c|}{ Golosinas } \\
\hline & Sí & No & Sí & No & Sí & No \\
\hline Siempre & $241(40,8 \%)$ & $350(59,2 \%)$ & $310(51,6 \%)$ & $291(48,4 \%)$ & $278(47,2 \%)$ & $311(52,8 \%)$ \\
\hline Nunca & $821(34,7 \%)$ & $1543(65,3 \%)$ & $1150(49,1 \%)$ & $1193(50,9 \%)$ & $1141(48,1 \%)$ & $1232(51,9 \%)$ \\
\hline A veces & $1628(35,7 \%)$ & $2919(64,2 \%)$ & $2239(49,5 \%)$ & $2285(50,5 \%)$ & $2564(55,8 \%)$ & $2030(44,2 \%)$ \\
\hline & \multicolumn{5}{|c|}{ Práctica de actividad física fuera del horario escolar } & \\
\hline Siempre & & & $444(70,3 \%)$ & & $186(29,4 \%)$ & \\
\hline Nunca & & & $835(74,6 \%)$ & & $622(25,3 \%)$ & \\
\hline A veces & & & $533(75,1 \%)$ & & $1.171(24,9 \%)$ & \\
\hline
\end{tabular}

\begin{tabular}{|lrrrrr|}
\hline & \multicolumn{3}{c}{ Tipo de establecimiento cercano al centro educativo frecuentado durante el recreo } \\
Quiosco & Cafetería & Supermercad & Tienda de Comestibles & Otros establecimientos \\
& & & o & & \\
Siempre & $\mathbf{1 5 1}(\mathbf{2 4 , 1 \% )}$ & $\mathbf{2 7 2}(\mathbf{4 4 , 4 \% )}$ & $\mathbf{7 1}(\mathbf{1 1 , 6 \% )}$ & $\mathbf{1 3 5}(\mathbf{2 2 \%})$ & $\mathbf{9 2}(\mathbf{1 5 \%})$ \\
A veces & $\mathbf{3 2 6}(\mathbf{1 3 , 5 \% )}$ & $\mathbf{3 6 7}(\mathbf{1 5 , 4 \% )}$ & $\mathbf{1 9 2}(\mathbf{8 \%})$ & $\mathbf{1 5 4}(\mathbf{6 , 5 \% )}$ & $\mathbf{8 9}(\mathbf{3 , 7 \%})$ \\
\hline
\end{tabular}

*TIPO DE DESAYUNO: TIPO 1: Desayuno completo con pan o cereal; TIPO 2: Desayuno completo con dulce; TIPO 3: Medio desayuno con pan y/o cereal; TIPO 4: Medio desayuno con dulce; TIPO 5: Medio desayuno con pan y dulce; TIPO 6: Desayuno sólido incompleto; TIPO 7: Desayuno líquido incompleto; TIPO 8: Desayuno de baja calidad nutricional; TIPO 9: Miscelánea de alimentos. **TIPO DE MERIENDA ESCOLAR: TIPO 1: Sólo alimentos líquidos; TIPO 2: Líquidos+Productos No Recomendables en una Alimentación Saludable (PNRAS); TIPO 3: Alimentos sólidos + PNRAS; TIPO 4: Alimentos sólidos + líquidos; TIPO 5: Alimentos sólidos + líquidos + fruta; TIPO 6: Alimentos sólidos + líquidos + PNRAS; TIPO 7: Sólo PNRAS; TIPO 8: Responde alimentos que consume habitualmente; Ns/Nc: No sabe, No contesta. 


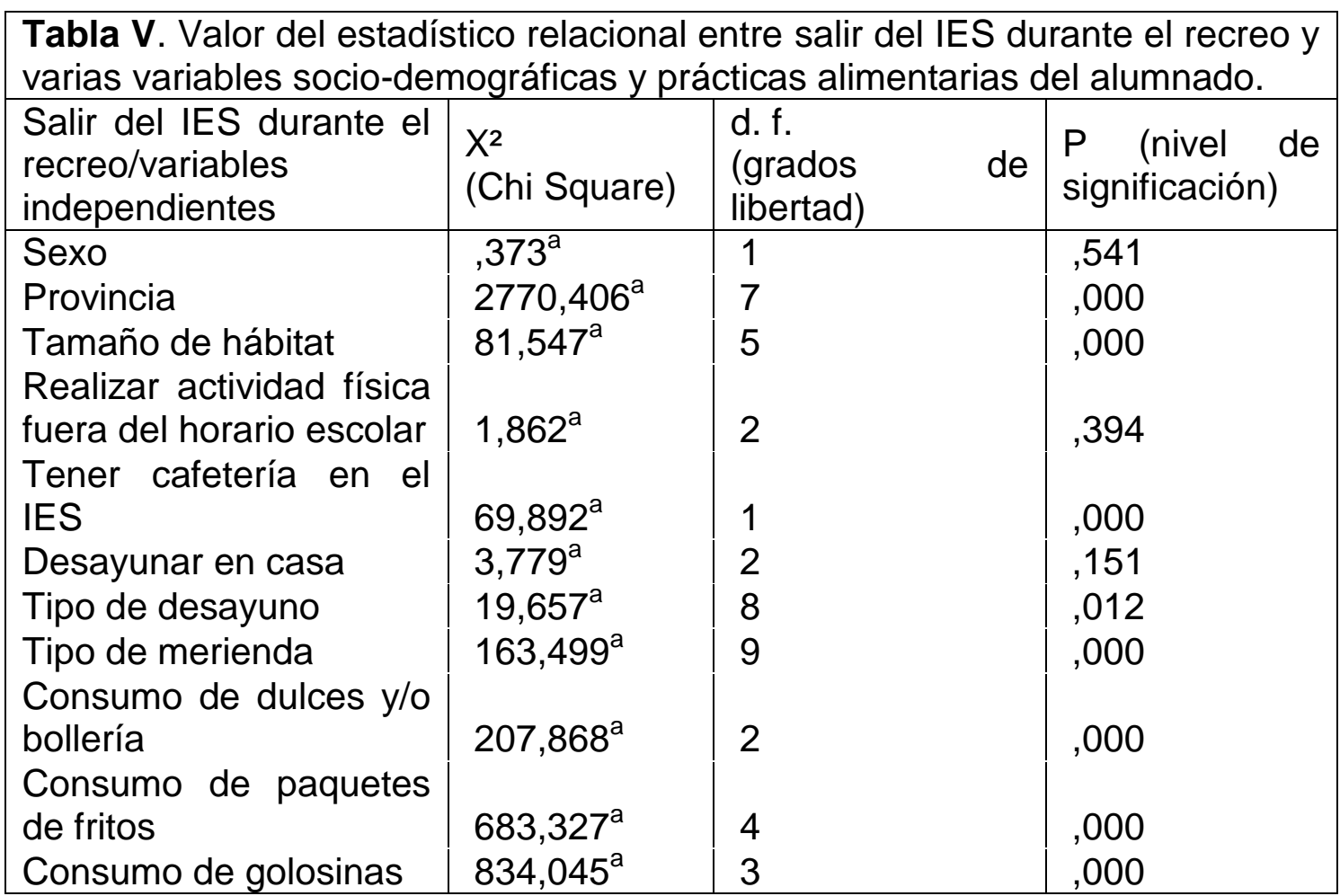

Finalmente destacar el mayor consumo de golosinas entre el alumnado que sale del centro durante el recreo $(71,4 \%)$ frente al que no $(65,5 \%)$, y el consumo esporádico de dulces y bollería mayor entre el alumnado que utiliza estos establecimientos durante la hora del recreo (76,5\%). Tanto en el grupo de alumnos/as que afirma consumir siempre Dulces y/o bollería y paquetes de fritos la frecuencia es mayor entre los que no salen del IES (14,1\% y $25,2 \%)$ que entre los que afirman salir a la hora de recreo $(5,5 \%$ y $5,8 \%)$, por lo que la existencia de estos locales externos no influye más que la oferta del propio centro.

\section{DISCUSIÓN}

Se constata un gran número de institutos de educación secundaria que cuentan en sus alrededores con establecimientos que ofertan productos alimentarios de baja calidad nutricional, disponibles además en el horario de la jornada escolar. Estos locales suelen ser utilizados por un cuarto del alumnado de secundaria andaluz muestreado durante la hora del recreo, de forma permanente pero con bastante más frecuencia de forma esporádica. Esta realidad, es coincidente con la encontrada por otros autores que han buscado conocer los elementos que aparecen en el entorno y que favorecen el consumo de alimentos de alta densidad calórica y baja calidad nutricional. En un estudio americano se identifican cuatro tipos de tiendas de comestibles en el entorno de los centros escolares ${ }^{12}$ : cadenas de supermercados, supermercados autónomos, tiendas de conveniencia y otras tiendas de comestibles; concluyendo que las tiendas de mayor superficie ofertan productos frescos y de mejor calidad nutricional frente a la oferta de otros establecimientos. Por otro lado, los resultados de otros estudios indican que la existencia de tiendas de conveniencia a una distancia de 10 minutos de las escuela -que en el contexto de nuestro estudio pueden ser comparables a las pequeñas tiendas de desavío- se asoció con una mayor tasa de estudiantes con sobrepeso, en comparación con los estudiantes de escuelas sin tiendas de estas características ${ }^{11}$. En el caso de nuestra investigación, tal como reflejan nuestros resultados, el tipo de establecimiento más frecuente en 
todas las provincias es el bar y la cafetería, hecho por el que debe ser el tipo de local más utilizado por el alumnado en estudio. Resulta llamativo que exista un número relevante de tiendas dedicadas a la venta de productos no saludables dirigidos especialmente a los estudiantes y alarmante, la existencia de quioscos dedicados a la venta exclusiva de chucherías. La provincia de Huelva es la que más centros educativos tiene con este tipo de comercio en sus cercanías frente a Jaén o Granada que presentan menos. Este hallazgo debe favorecer el que el alumnado onubense sea uno de los que más sale fuera del centro durante el recreo. Aunque en nuestro investigación no se ha estudiado la relación con la tasa de sobrepeso, sí podemos concluir que el predominio de tiendas pequeñas y quioscos, cuyo potencial de ofrecer productos no recomendados en una dieta saludable es muy elevado, ponen de relieve el alto riesgo para la seguridad alimentaria del alumnado de secundaria andaluz que convive en un entorno escolar poco saludable. Con independencia de las agrupaciones de los comercios, la oferta de chucherías se encuentra en todas las tipologías de establecimientos, aunque con menor intensidad en el sector 2 que aglutina a bares y cafeterías y, la sección 3, dedicada a la venta de churros y patatas fritas exclusivamente.

Lo que empeora aún más la calidad del ambiente, sin control por la administración central y sin una política de promoción de la alimentación saludable desde la administración local. Este suceso parece influir en el mayor consumo de golosinas del alumnado que sale durante el recreo del centro según la relación significativa encontrada. No ocurre lo mismo con el consumo de bollería y paquetes de fritos debido, tal vez, a que la oferta interna del centro sea en estos últimos casos tan elevada ${ }^{19}$ o similar a la externa, lo que no le obliga a salir para adquirirlos.

Respecto a los resultados encontrados en el resto de prácticas alimentarias del alumnado durante la jornada escolar, podemos concluir que el alumnado que se ausenta durante el recreo posee unos hábitos de desayuno de peor calidad frente al grupo de alumnos/as que permanece en el centro, aunque este hecho puede estar relacionado con otros factores sociales que condicionan las prácticas de los adolescentes. La existencia de estos establecimientos por su accesibilidad en el entorno cotidiano del alumnado se configura claramente como un factor que puede favorecer elecciones alimentarias poco saludables y que compiten con las prácticas alimentarias familiares tradicionales.

Otros de los hallazgos encontrados en nuestro estudio, respecto a la calidad de la oferta alimentaria, está relacionada con la ausencia de pan tradicional en la elaboración de los bocadillos. La penetración en el mercado de pan precocinado se da en todos los sectores de consumo y se constata en este ámbito ${ }^{18}$.

Los métodos utilizados para establecer la distancia entre los centros escolares y los establecimientos que ofertan comestibles es diversa. En algunos se utiliza el tiempo ${ }^{11}$, pero no se han encontrado estudios que hayan medido la distancia en pasos desde los centros de enseñanza secundaria y los comercios de comestibles para su comparación. En Andalucía, mayoritariamente los locales están ubicados a escasa distancia de los centros educativos, con cierta variabilidad entre las provincias. Cádiz y Córdoba concentran el mayor número de locales con mayor proximidad. Por el contrario Jaén y Málaga se encuentran más distanciados. Huelva sería por número de establecimientos y cercanía, la provincia que posee un entorno de mayor riesgo respecto a la disponibilidad de productos no recomendados en la alimentación saludable. Este hecho podría asociarse con el estudio que indica que el alumnado 
onubense es el mayor consumidor de estos productos respecto a los estudiantes de otras provincias ${ }^{1}$.

A pesar de que la existencia de estos establecimientos es conocida por parte de los responsables directivos de los centros estudiados, no se identifica como un signo de alarma o no se considera un tema preocupante ya que al encontrarse fuera del recinto escolar se aleja de su responsabilidad.

Es evidente que las intervenciones ambientales eficaces, que combatan la pandemia de obesidad, deben facilitar y apoyar las decisiones más saludables en el alumnado ${ }^{6}$. En este sentido cualquier estrategia dirigida a las instituciones debería contemplar el incentivar el consumo de fruta entre los escolares fomentando la accesibilidad de estos productos en el entorno escolar y extraescolar.

Como líneas futuras de investigación, consideramos que los estudios deberían analizar los hábitos alimentarios extra-domésticos de los escolares, especialmente aquellas prácticas y elecciones alimentarias ligadas al entorno extraescolar; y aumentar los estudios dedicados al conocimiento de las características del entorno cercano de los escolares para determinar qué factores ambientales están actuando, cuáles pueden incidir negativamente y cuáles pueden favorecer prácticas alimentarias saludables. Igualmente, sería interesante que en estudios sucesivos, no sólo se analizara el consumo externo durante el recreo, sino que éste se ampliara también a la adquisición de productos alimentarios en estos locales durante el traslado matutino de casa al IES. En este estudio no se ha contemplado y sin lugar a dudas, hubiera incrementado la frecuencia de utilización de estos establecimientos.

\section{Agradecimientos}

A la Confederación Andaluza de Asociaciones de Padres y Madres de alumnos/as por una educación pública de calidad (CODAPA) por la petición de realizar este estudio y por el apoyo mostrado durante el mismo.

\section{REFERENCIAS}

1. García Padilla FM, González Rodríguez A, Martos Cerezuela I y González Delgado A. Perfil alimentario del alumnado de secundaria en Andalucía durante la jornada escolar. Nutr clín diet hosp 2013; 33: 129

2. Palenzuela Paniagua SM, Pérez Milena A, Pérula de Torres LA, Fernández García JA y Maldonado Alconada J. La alimentación en el adolescente. An Sist Sanit Navar 2014; 37: 47-58

3. Francis LA, Lee $Y$ y Birch LL. Parental weight status and girls' television Beijing, snacking, and body mass indexes. Obes Res clin Pract 2003;11: 143. Disponible en: http://www.ncbi.nlm.nih.gov/pmc/articles/PMC2530922/

4. Healthy Lifestyle in Europe by Nutrition in adolescente. Disponible en: www.helenastudy.com

5. Black J, y Macinko J. Neighborhoods and obesity. Nutr Rev 2007; 66: 2-20. doi:10.1111/j.1753-4887.2007.00001.x

6. Lake A y Townshend T. Obesogenic environments: exploring the built and food environments. JRSH 2006; 126: 262.Disponible en: http://rsh.sagepub.com/content/126/6/262

7. Feng J, Glass TA, Curriero FC, Stewart WF y Shwartz BS. The built environment and obesity: A systematic review of the epidemiologic evidence. Health Place 2010; 16: 175 
8. Fleischhacker SE, Evenson KR, Rodríguez DA y Ammerman AS. A systematic review of fast food access studies. Obes Rev 2010; 12: 460.

Doi: 10.1111/j.1467-789X.2010.00715.x

9. Richardson AS, Boone-Heinonen J, Popkin BM y Gordon-Lansen P. Neighborhood fast food restaurants and fast food consumption: A national study. BMC Public Health 2011; 11:543. Disponible en: http://www.biomedcentral.com/14712458/11/543

10. Story M, Kaphingst KM, Robinson-O’Brien R y Glanz K. Eating Environments: Policy and Environmental Approaches. Annu Rev Public Health 2008; 29: 253

11. Howard PH, Fitzpatrick M y Fulfrost B. Proximity of food retailers to schools and rates of overweight ninth grade students: an ecological study in California. BMC Public Health 2011; 11:68. Disponible en: http://www.biomedcentral.com/14712458/11/68

12. Powell LM, Auld MC, Chaloupka FJ, O’Malley PM y Johnston LD. Associations Between Access to Food Stores and Adolescent Body Mass Index. Am J Prev Med 2007; 33: 301

13. Ahrens W, Bammann K, Siani A, Buchecker K, De Henauw S, lacoviello L et al The IDEFICS cohort: design, characteristics and participation in the baseline survey. Int J Obes 2011; 35: S3-S15.

14. Buck C, Börnhorst C, Pohlabeln H, Huybrechts I, Pala V, Reisch L, et al. Clustering of unhealthy food around German schools and its influence on dietary behavior in school children: a pilot study. Int J Behav Nutr Phys Act 2013; 10:65. doi:10.1186/1479-5868-10-65

15. Engler-Stringer R, Le H, Gerrard A y Muhajarin N. The community and consumer food environment and children's diet: a systematic review. BMC Public Health 2014; 14:522. doi:10.1186/1471-2458-14-522

16. Boletín Oficial del Estado. Ley $17 / 2011$, de 5 de julio, de seguridad alimentaria y nutrición. BOE núm 160 de 6/7/2011.

17. González Rodríguez A, García Padilla FMaㅡ, Liébana Fernández JL, Delgado de Mendoza Núñez J, González de Haro MD, Silvano Arranz A et al. La ley de Seguridad alimentaria y nutrición en los centros de educación secundaria andaluces. Gac Sanit 2013; 27: 357

18. Gil A, Serra L. Libro blanco del pan. Madrid: Médica Panamericana; 2010.

19. González Rodríguez A, García Padilla FMaa, Martos Cerezuela I, Silvano Arranz A, Fernández Lao I, et. al. PROYECTO ANDALIES: Consumo, oferta y promoción de la alimentación saludable en los centros de educación secundaria de Andalucía. Nutr Hosp. 2015; 31(4): 1853-1862

\footnotetext{
${ }^{\wedge}$ En esta investigación han participado además: Marta de Miguel González, José Luís Puentes Carmena, Román Puentes Sánchez, Miguel Ramón Camacho, Pilar Torres Alegre, Jesús Delgado de Mendoza Núñez, Mํㅡ Luisa Castilla Romero, Ascensión Delgado Romero, Jesús Fernández Rodríguez, Ana de Irigoyen Diaz, José Manuel Martínez Nieto, Monsalud Moreno García, Cristina Delgado de Mendoza Manzano, Antonio González Delgado, Esteban García Lara, José Luís Gutiérrez Sequera, Antonia Martínez Orozco, Inmaculada Poyato Luque, Carmen Rodríguez Palma, Ana Salinas Lozano, Feliciano Santos Blanco, Ma José Utrero García, Alejandro Granados Alba, Miguel Angel Castro Turrión, Gracia Rosa García Martín, Nieves Lafuente Robles, Francisco Javier Muñoz Rodríguez, Lucía Palomares Ojeda, Juan Luis Vilchez Melgarejo, Mạ Dolores González de Haro, Pepa Frigolet Maceras, José Luís Liébana Fernández, Mª Aurora Quero Cabeza, Jesús Luque Peña, Josefina Arboledas Bellón, Inmaculada Fernández Guillén, Matilde Pumarejo Rosado, Eusebia Requena Fernández, Reme Rojano Pérez, Asunción Uli Elizalde, Mํa José Sánchez Cordero, Ángela Cejudo López, Juan Antonio Melo Salor
}

Recibido: 18 de octubre 2015;

Aceptado: 18 de diciembre 2015 